\title{
Early enteral feeding strategies for very preterm infants: current evidence from Cochrane reviews
}

\author{
The SIFT Investigators Group
}

\section{NECROTISING ENTEROCOLITIS AND LATE-ONSET INFECTION}

Although antenatal and neonatal interventions and care practices have increased survival and improved long-term outcomes for very preterm ( $<32$ weeks gestation) or very low birthweight (VLBW: <1500 g) infants, the incidence of necrotising enterocolitis (NEC) and late-onset (nosocomial) infection remains high. Late-onset infection affects about $20 \%$ and NEC occurs in about $5 \%$ of all of very preterm or VLBW infants. ${ }^{12}$ Attributable mortality is high (>20\%), especially for severe NEC and gram-negative bacillus or fungal infection. ${ }^{3}$ These conditions are now responsible for more deaths beyond the early neonatal period than any other causes. ${ }^{5} 6$ NEC and late-onset infection are also associated with important morbidities including reduced nutrient intake and slow growth, a longer duration of intensive care and hospital stay, and a higher incidence of long-term neurological disability $^{7-9}$ (figure 1).

\section{INTRODUCING AND ADVANCING ENTERAL FEEDS}

The timing of introduction and the rate of advancement of enteral milk feeds for very preterm or VLBW infants has the potential to influence important outcomes including the risk of NEC and late-onset infection. Observational studies have suggested that delaying the introduction of progressive enteral feeding until about 57 days after birth and increasing the volume of milk feeds slowly $(<24 \mathrm{ml} / \mathrm{kg}$ ) day) is associated with a lower risk of developing NEC. ${ }^{10}{ }^{11}$ However, there are also potential disadvantages associated with conservative enteral feeding regimens. Delayed or slow enteral feeding may diminish the functional adaptation of the gastrointestinal tract and disrupt the patterns of microbial colonisation. ${ }^{12} 13$ Intestinal dysmotility may exacerbate feed intolerance leading to a delay in establishing enteral feeding independently of

Correspondence to Professor William McGuire, NIHR Centre for Reviews and Dissemination, University of York, York, Y010 5DD, UK; william.mcguire@hyms.ac.uk parenteral nutrition. Prolonging the duration of parenteral nutrition may be associated with infectious and metabolic complications that increase mortality and morbidity, prolong hospital stay, and adversely affect growth and development. ${ }^{14} 15$

\section{EVIDENCE-BASE FOR EARLY ENTERAL FEEDING STRATEGIES}

Given the potential for early enteral feeding strategies to affect important outcomes, it is essential that policy and practice is based on the best quality evidence possible. Currently, wide variation in policy and practice exists internationally, and between and within neonatal units. ${ }^{16}$ Observational data may be confounded by known and unknown factors, for example, clinician preferences or other care practices, that affect outcomes independently of the feeding method. Adequately powered randomised controlled trials obviate these issues and provide the least biased assessment of the impact of different feeding methods. Cochrane systematic reviews seek to identify and appraise randomised trials to provide a synthesised summary of the evidence. Three Cochrane reviews of enteral feeding strategies focus on distinct clinical scenarios. ${ }^{17-19}$

\section{EARLY TROPHIC FEEDING VERSUS ENTERAL FASTING}

Early trophic feeding is conventionally defined as giving small volumes of milk (typically $12-24 \mathrm{ml} / \mathrm{kg} /$ day) without advancing the feed volumes during the first five to seven postnatal days. ${ }^{20}$ The primary aim is to accelerate gastrointestinal physiological, endocrine and metabolic maturity and so allow infants to transition to full enteral feeding independent of parenteral nutrition more quickly.

The Cochrane review of trophic feeding versus enteral fasting for very preterm or VLBW infants includes nine trials in which a total of 754 infants participated. ${ }^{17}$ Few participants were extremely preterm ( $<28$ weeks) or extremely low birthweight (ELBW: $<1000 \mathrm{~g}$ ) or growth restricted. None of the trials specifically recruited infants with absent or reversed end- diastolic flow velocities on antenatal Doppler studies.

These trials did not provide evidence that early trophic feeding affected feed tolerance or growth rates. Although some trials reported that trophic feeding reduced the time taken to establish full enteral feeds, meta-analysis of all of the available data did not detect a statistically significant effect. The trial data do not suggest that early trophic feeding is associated with important harms. Meta-analyses did not detect statistically significant effects on the incidence of NEC, late-onset infection or all-cause mortality (figures 2 and 3). The trials found inconsistent effects on short-term growth and meta-analysis did not reveal a significant difference in the time taken to regain birthweight.

One trial reported that mothers who expressed breast milk for early trophic feeding were more likely to continue to provide breast milk as the ongoing principal form of nutrition for their infants. ${ }^{21}$ Further study to confirm and define the mechanism of this association is merited given that feeding with breast milk compared to formula reduces the risk of NEC in very preterm or VLBW infants. ${ }^{22}$

\section{Delayed versus early introduction of} progressive enteral feeds

The Cochrane review of delayed versus early introduction of progressive enteral feeding identified seven randomised controlled trials in which a total of 964 infants participated. ${ }^{18}$ The trials defined delayed introduction as later than 5-7 days after birth and early introduction as up to 4 days after birth. Meta-analyses did not detect statistically significant effects on the risk of NEC or all-cause mortality (figures 2 and 3). Three of the trials (including a recent, large, UK and Ireland 54-centre trial) restricted participation to growth-restricted infants with Doppler ultrasound evidence of abnormal fetal circulatory distribution or flow. ${ }^{23}$

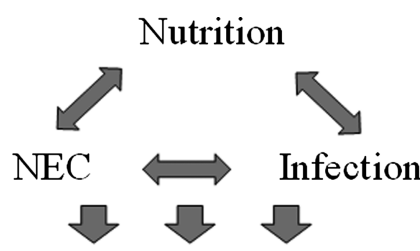

\section{Mortality and morbidity \\ Growth and development}

Figure 1 Nutrition, necrotising enterocolitis and late-onset infection affect important outcomes. 


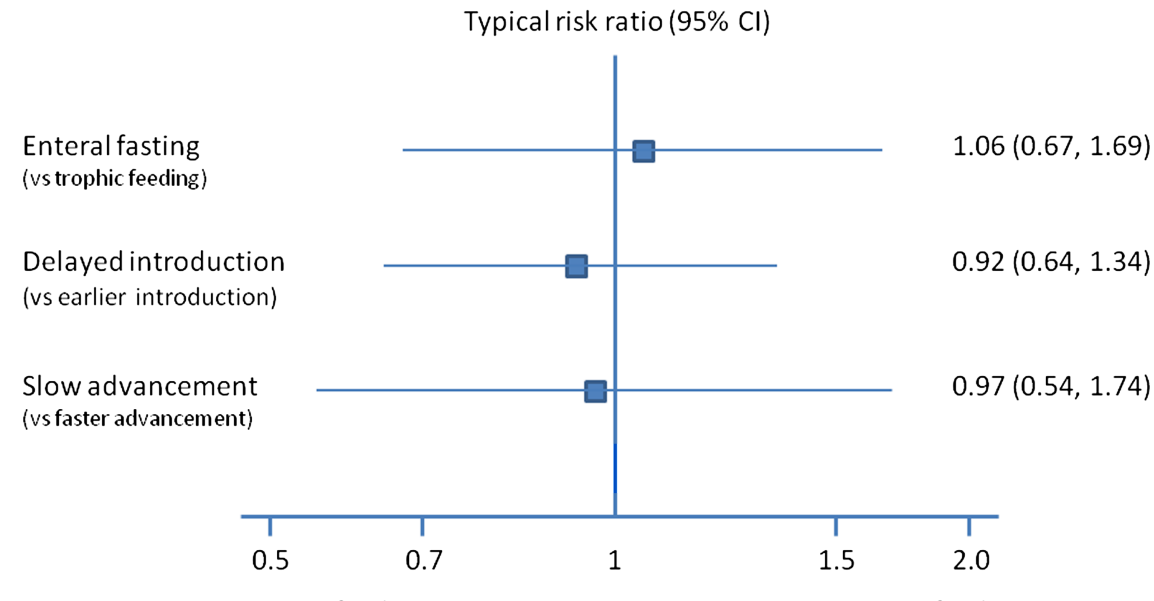

Favours conservative feeding regimen

Favours progressive feeding regimen

Figure 2 Summary meta-analyses of conservative versus progressive early enteral feeding: incidence of necrotising enterocolitis.

Planned subgroup analyses of these trials did not find any statistically significant effects on the risk of NEC or all-cause mortality. Infants who had delayed introduction of enteral feeds took longer to establish full enteral feeding (median difference 2 to 4 days). It is not yet known whether this is associated with important clinically adverse consequences such as a higher rate of late-onset infection secondary to prolonged use of parenteral nutrition or a longer duration of hospital admission.

\section{Slow versus faster advancement of enteral feed volumes}

The Cochrane review identified five randomised controlled trials in which a total of 588 infants participated. ${ }^{19}$ Few participants were extremely preterm, ELBW or growth restricted. The trials defined slow advancement as daily increments of infection or the duration of hospital stay.

\section{LIMITATIONS OF EVIDENCE}

$15-20 \mathrm{ml} / \mathrm{kg}$ and faster advancement as $30-35 \mathrm{ml} / \mathrm{kg}$. Meta-analyses did not detect statistically significant effects on the risk of NEC or all-cause mortality (figures 2 and 3). Infants who had slow advancement took significantly longer to regain birthweight (median differences 2 to 6 days) and to establish full enteral feeding (2-5 days). The trial data did not provide evidence of an effect on the incidence of late-onset

The randomised controlled trials included in these Cochrane reviews were generally of good methodological quality but none of the trials masked caregivers and clinical assessors to the nature of the intervention. Although the lack of blinding may have resulted in surveillance and ascertainment biases, this is more likely to have caused an overestimation of the incidence of

Typical risk ratio $(95 \% \mathrm{Cl})$

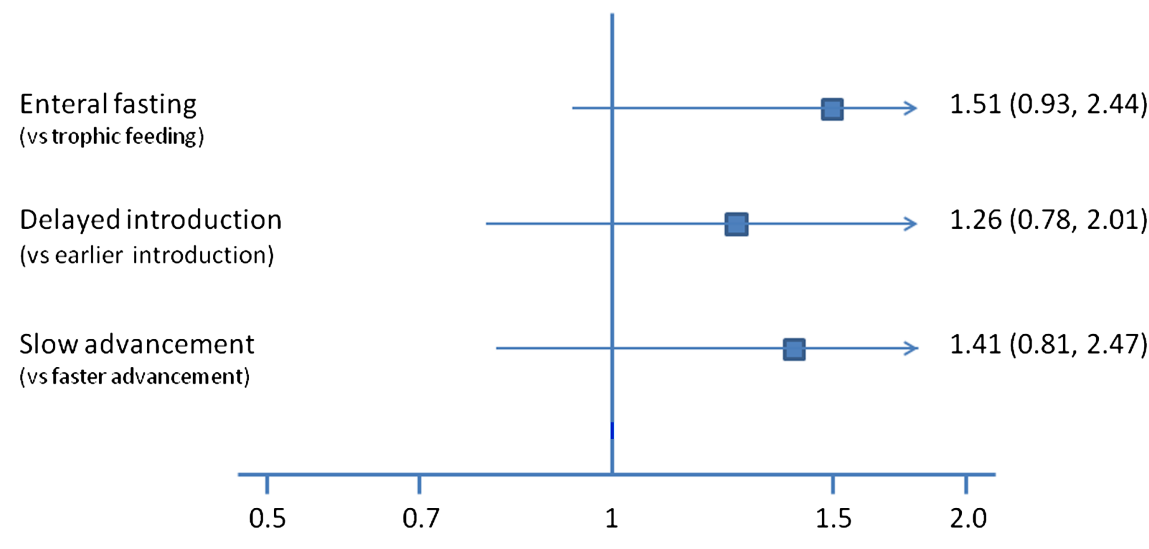

Favours conservative feeding regimen

Favours progressive feeding regimen

Figure 3 Summary meta-analyses of conservative versus progressive early enteral feeding: all-cause mortality.
NEC in infants whose feed volumes were introduced earlier or advanced faster. The assessment of abdominal radiographs for signs of NEC was masked in most trials to ensure that the diagnosis of severe NEC (confirmed by the radiological detection of gas in the bowel wall or portal tract) was not prone to bias. However, since the microbial generation of gas in the bowel wall is substrate-dependent, infants who received more enteral milk (substrate) may have been more likely to demonstrate this radiological sign than infants with equally severe bowel disease who had received less milk. This 'substrate effect' is also more likely to cause over-ascertainment of NEC in the infants who had faster rates of feed volume advancement. ${ }^{24}$

\section{IMPLICATIONS FOR PRACTICE}

The available trial data suggest that introducing progressive enteral feeding before 4 days after birth and advancing the rate of feed volumes at more than $24 \mathrm{ml} / \mathrm{kg}$ / day does not increase the risk of NEC in very preterm or VLBW infants. These findings are consistent with policy and practice in some countries, notably in Scandinavia, where very early introduction and advancement of enteral feeds (often within 24 to $48 \mathrm{~h}$ after birth) has not been associated with a higher incidence of NEC. ${ }^{1625}$ Delayed introduction or slow advancement results in several days of delay in the time taken to regain birthweight and establish full enteral feeds. The long-term clinical importance of these effects is unclear. However, the generalisability of these data for extremely preterm or ELBW infants is unclear as this group contributed only a minority of the total participants in the existing trials. Uncertainty also exists about the riskbenefit balance of different enteral feeding strategies in human milk-fed versus formula-fed very preterm or VLBW infants as the trials and reviews did not contain sufficient data for subgroup analyses.

\section{IMPLICATIONS FOR RESEARCH}

Further randomised controlled trials could provide more precise estimates of the effects of early enteral feeding on important outcomes for very preterm or VLBW infants. Trials should aim to ensure the participation of ELBW and extremely preterm infants as well as infants with evidence of compromised intrauterine growth so that subgroup analyses can be planned for these infants at highest risk of NEC. Masking caregivers and investigators to these interventions is unlikely to be possible. Since the unblinded 
evaluation of NEC and late-onset infection is subject to surveillance and ascertainment biases, trials should aim to assess more objective outcomes, principally allcause mortality and long-term growth and development.

SIFT

The success of the large 'Antenatal Doppler Enteral Prescription Trial' (ADEPT) in assessing the effect of delayed versus early (within $48 \mathrm{~h}$ of birth) enteral feeding for growth-restricted infants has generated interest and enthusiasm for further trials to assess enteral feeding strategies in very preterm or VLBW infants. In the UK and Ireland, the 'Speed of Increasing Feeds Trial' (SIFT) Group, a collaboration of service-user representatives, clinicians and trial unit experts, is undertaking a pragmatic randomised controlled trial in which 2500 very preterm or VLBW infants will be enrolled. The trial will compare advancing enteral feeds at either $30 \mathrm{ml} / \mathrm{kg} /$ day or $18 \mathrm{ml} / \mathrm{kg} /$ day. To enhance generalisability, human milk-fed and formula-fed infants will be eligible to participate and daily feeding logs will record the type of milk given. The primary outcome is death or moderate or severe disability at 2 years post-term and analyses will be by intention-to-treat. The trial is also powered to assess meaningful effects on in-hospital mortality and major morbidity, antibiotic usage and duration of hospital stay. We will conduct an economic evaluation to assess whether the intervention is likely to be cost-effective. SIFT is designed to run in parallel with another large UK multi-centre trial (ELFIN) that aims to assess the effect of prophylactic enteral lactoferrin supplementation for very preterm infants. ${ }^{26}$

Collaborators The SIFT Investigators Group [J Abbott, JE Berrington, E Boyle, JS Dorling, NE Embleton, E Juszczak, AA Leaf, L Linsell, $S$ Johnson, $K$ McCormick, W McGuire, T Roberts, B Stenson].

\section{Competing interests None.}

Provenance and peer review Commissioned; externally peer reviewed.

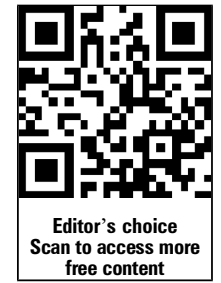

To cite The SIFT Investigators Group. Arch Dis Child Fetal Neonatal Ed 2013;98:F470-F472.

Received 14 March 2013

Revised 29 April 2013

Accepted 9 May 2013

Published Online First 13 June 2013

Arch Dis Child Fetal Neonatal Ed 2013;98:F470-F472. doi:10.1136/archdischild-2012-303260

\section{REFERENCES}

1 Vergnano S, Menson E, Kennea N, et al. Neonatal infections in England: the NeonIN surveillance network. Arch Dis Child Fetal Neonatal Ed 2011;96: F9-14.

2 Morgan JA, Young L, McGuire W. Pathogenesis and prevention of necrotizing enterocolitis. Curr Opin Infect Dis 2011;24:183-9.

3 Samanta S, Farrer K, Breathnach A, et al. Risk factors for late onset gram-negative infections: a case-control study. Arch Dis Child Fetal Neonatal Ed 2011;96:F15-18.

4 Rees CM, Eaton S, Pierro A. Trends in infant mortality from necrotizing enterocolitis in England and Wales and the USA. Arch Dis Child Fetal Neonatal Ed 2008;93:F395-6.

5 Horbar JD, Carpenter JH, Badger GJ, et al. Mortality and neonatal morbidity among infants 501 to 1500 grams from 2000 to 2009. Pediatrics 2012;129:1019-26.

6 Berrington JE, Hearn RI, Bythell $M$, et al. Deaths in preterm infants: changing pathology over 2 decades. J Pediatr 2012;160:49-53.

7 Stoll BJ, Hansen NI, Adams-Chapman I, et al. Neurodevelopmental and growth impairment among extremely low-birth-weight infants with neonatal infection. JAMA 2004;292:2357-65.

8 Pike $K$, Brocklehurst $P$, Jones $D$, et al. Outcomes at 7 years for babies who developed neonatal necrotising enterocolitis: the ORACLE Children Study. Arch Dis Child Fetal Neonatal Ed 2012;97:F318-22.

9 Bassler D, Stoll BJ, Schmidt B, et al. Using a count of neonatal morbidities to predict poor outcome in extremely low birth weight infants: added role of neonatal infection. Pediatrics 2009;123:313-18.

10 Henderson G, Craig S, Brocklehurst $P$, et al. Enteral feeding regimens and necrotising enterocolitis in preterm infants: a multicentre case-control study. Arch Dis Child Fetal Neonatal Ed 2009;94:F120-3.

11 Hartel C, Haase B, Browning-Carmo K, et al. Does the enteral feeding advancement affect short-term outcomes in very low birth weight infants? J Pediatr Gastroenterol Nutr 2009;48:464-70.

12 Stewart CJ, Nelson A, Scribbins D, et al. Bacterial and fungal viability in the preterm gut: NEC and sepsis. Arch Dis Child Fetal Neonatal Ed 2013;98: F298-F303.

13 Berrington JE, Stewart CJ, Embleton ND, et al. Gut microbiota in preterm infants: assessment and relevance to health and disease. Arch Dis Child Fetal Neonatal Ed 2013:98:F286-90.

14 Rønnestad A, Abrahamsen TG, Medbø S, et al. Late-onset septicemia in a Norwegian national cohort of extremely premature infants receiving very early full human milk feeding. Pediatrics 2005;115: e269-76.

15 Rees CM, Pierro A, Eaton S. Neurodevelopmental outcomes of neonates with medically and surgically treated necrotizing enterocolitis. Arch Dis Child Fetal Neonatal Ed 2007:92:F193-8.

16 Klingenberg C, Embleton ND, Jacobs SE, et al. Enteral feeding practices in very preterm infants: an international survey. Arch Dis Child Fetal Neonatal Ed 2012;97:F56-61.

17 Morgan J, Bombell S, McGuire W. Early trophic feeding versus enteral fasting for very preterm or very low birth weight infants. Cochrane Database Syst Rev 2013;3:CD000504.

18 Morgan J, Young L, McGuire W. Delayed introduction of progressive enteral feeds to prevent necrotizing enterocolitis in very low birth weight infants. Cochrane Database Syst Rev 2013;5: CD001970.

19 Morgan J, Young L, McGuire W. Slow advancement of enteral feed volumes to prevent necrotizing enterocolitis in very low birth weight infants. Cochrane Database Syst Rev 2013;3:CD001241.

20 McClure RJ. Trophic feeding of the preterm infant. Acta Paediatrica 2001:90:19-21.

21 Schanler RJ, Shulman RJ, Lau C, et al. Feeding strategies for premature infants: randomized trial of gastrointestinal priming and tube-feeding method. Pediatrics 1999;103:434-9.

22 Quigley MA, Henderson G, Anthony MY, et al. Formula milk versus donor breast milk for feeding preterm or low birth weight infants. Cochrane Database Syst Rev 2007;4:CD002971.

23 Leaf A, Dorling J, Kempley S, et al., Abnormal Doppler Enteral Prescription Trial Collaborative Group. Early or delayed enteral feeding for preterm growth-restricted infants: a randomized trial. Pediatrics 2012;129:e1260-8.

24 Tyson JE, Kennedy KA, Lucke JF, et al. Dilemmas initiating enteral feedings in high risk infants: how can they be resolved? Semin Perinatol 2007;31:61-73.

25 Fellman V, Hellström-Westas L, et al. EXPRESS Group. One-year survival of extremely preterm infants after active perinatal care in Sweden. JAMA 2009;301:2225-33.

26 ELFIN Trial Investigators Group. Lactoferrin immunoprophylaxis for very preterm infants. Arch Dis Child Fetal Neonatal Ed 2013;98:F2-4. 